\title{
Discrete Event Simulation and Petri net Modeling for Reliability Analysis
}

\author{
${ }^{1 *}$ Behrouz Safarinejadian \\ ${ }^{1}$ Shiraz University of Technology, safarinejad@sutech.ac.ir
}

\begin{abstract}
Analytical methods in reliability analysis are useful for studying simple problems. For complex networks with cross-linked (non-series/parallel) component configurations, it is difficult to use mathematical reliability analysis. Powerful methods for reliability analysis of such systems have been developed using discrete event simulation. The main drawback of these methods is that they are computer time intensive. In this paper, the main idea behind these methods is further explored and modified in order to reduce the computational loads. The modified approach presented here leads to a great time saving which is very important for reliability analysis of large scale systems. This modified method is then modeled by Petri net, which is a powerful modeling tool. The network reliability modeling technique developed in the paper has two main advantages. First, it can be easily implemented through a systematic and standard approach. Second, the developed model will greatly help solving the reliability analysis problem since it is simple and graphical.
\end{abstract}

Keywords: Reliability, discrete event simulation, Monte Carlo simulation, Petri net modeling

\section{Introduction}

Analytical methods in reliability analysis [1]-[5], are useful for studying simple problems in which severe limitations are placed on the distributional forms of the statistical data. Complex real world networks with their varied and poorly behaved component time-between-failure (TBF) and time-torepair (TTR) distributions and their cross-linked (non-series/parallel) component configurations, along with the desire to know the reliability/availability or TBF and TTR distributions at several points in the system, preclude the use of mathematical reliability/availability analyses. Hence, in complex systems such as electrical power, telephone, gas, water, and electro/mechanical systems, determining system reliability/availability or TBF and TTR distributions is done empirically if at all. In consequence, we need to study on new methods of reliability analysis. Our research objective is to propose new numerical methods of reliability analysis for large-scale networks.

Numerical methods of analysis are useful for studying complex problems [6]. In [7], two approaches are developed for the purpose of determining TBF and TTR distributions at various points in a system: (1) failure-mode method, and (2) flow method. These methods are very powerful and valuable since using these methods reliability of any large-scale network can be studied and there is no constraint on the network structure or reliability properties of the units. The main drawback of these methods is that they are computer time intensive. Here, these methods are further explored and are modified in order to reduce the computational load. The modification leads to a great time saving which is really important in reliability analysis of large systems.

In the methods reported here discrete simulation modeling is used to determine the TBF and TTR distributions at any point in a real world or hypothesized system using any kind of component TBF and TTR distributions. The model size and running time is essentially proportional to the number of components. The TBF and TTR are chosen as the measures of interest, rather than reliability/ 
International Journal of

Soft Computing And Software Engineering (JSCSE)

e-ISSN: 2251-7545

Vol.2,No.5, 2012

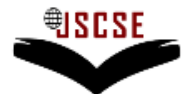

DOI: 10.7321/jscse.v2.n5.3

availability, because they are easier to understand by most users in these industries.

Then using Petri net, a discrete event modeling tool, [8]-[14], a modeling approach for the modified flow method has been developed. The network reliability modeling technique which is expressed here has two main advantages: First, using this technique, one can implement the modified flow method through a systematic and standard approach. Second, since the developed model is simple and graphical, it would help solving the reliability analysis problem.

The rest of the paper is organized as follows. Section 2 presents reliability analysis and then describes the methods of failure mod and flow. The modified versions of these two methods are developed in section 3. Section 4 is devoted for developing a modeling approach for the modified flow method using Petri net. Finally, section 5 concludes the paper.

\section{Reliability Analysis Using Monte Carlo Simulation}

In reliability analysis we will need some notation and definitions which express statistical properties of the units. TBF (time before fail) and TTR (time to repair) are two important random variables describing the statistical properties of the units' failure and repair. Here, these variables are briefly introduced.

The period of time before a unit fails is regarded as a nonnegative random variable named TBF and is denoted by $\zeta$. The statistical properties of random variable TBF is given through its distribution function [5]:

$$
F(t)=P\{\zeta \leq t\}
$$

This random variable can also be described by the following density function:

$$
f(t)=\frac{d}{d t} F(t)
$$

Reliability function is also defined as:

$$
R(t)=1-F(t)=P\{\zeta>t\}
$$

Another important factor is MTBF (mean time before fail) which is denoted by $T$ and is defined as:

$$
T=\int_{0}^{\infty} t f(t) d t
$$

The random variables TTR and MTTR (mean time to repair) are also defined similarly.

The important problem is how to study statistical reliability properties of a system according to the reliability properties of its units. Although analytical methods of reliability analysis have been used for this purpose, these methods are only applicable to a network with either series or parallel component configurations. Otherwise analytical methods may not be used and we need other more general approaches.

Monte Carlo simulation is used to study complex systems when analysis is difficult or impossible through analytical methods. In fact, Monte Carlo simulation is a statistical method for reliability analysis. Simulation results are assumed to be from a real system or field tests and statistical properties of these results are determined. A discrete event model is used for reliability assessment in which a sequence of events and their relations are modeled. Here, two Monte Carlo based simulation methods are explained for reliability analysis of a large scale system. In these simulation methods, as in mathematical reliability analysis, it is necessary to know beforehand (1) the statistical distributions of TBF and TTR for each component, and (2) the component configuration.

The hydraulic network shown in Figure 1 will be used to demonstrate the methodologies. Hydraulic network is a well known test bench that has also been used in previous studies [7] due to its simplicity and wide applications. The hydraulic network is successful as long as fluid can flow through 
International Journal of

Soft Computing And Software Engineering (JSCSE)

e-ISSN: 2251-7545

Vol.2,No.5, 2012

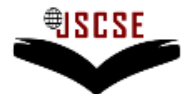

DOI: 10.7321/jscse.v2.n5.3

some pumps from left to right. The system fails if (1) both $A$ and $B$ fail; (2) X, Y and Z fail; (3) X, Y and $B$ fail; or (4) Y, Z, and $A$ fail (i.e., four minimum cut-sets). Assume the piping is $100 \%$ reliable. This network could just as well have represented as an electrical network with the components being substations or electronic devices and the pipes being wires.

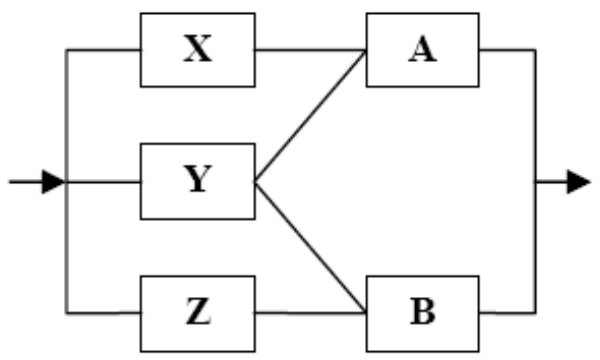

Figure 1. Cross-linked hydraulic network.

We will find the MTBF (mean-time-between-failures) and MTTR (mean-time-to-repair) and associated distributions of the output of the system if the TBF and TTR for the individual pumps are exponentially distributed and the MTBF and MTTR have the following values:

Table 1. MTBF and MTTR of the pumps.

\begin{tabular}{|c|c|c|}
\hline PUMP & MTBF & MTTR \\
\hline X, $\mathrm{Z}$ & $1000 \mathrm{~h}$ & $100 \mathrm{~h}$ \\
\hline $\mathrm{Y}$ & $500 \mathrm{~h}$ & $100 \mathrm{~h}$ \\
\hline A, B & $1200 \mathrm{~h}$ & $100 \mathrm{~h}$ \\
\hline
\end{tabular}

In failure mode method one should first identify all modes of system failure, i.e., list all subgroups of components that must fail for a system failure to occur. For example, in the cross-linked problem, if all the components in one of the following minimum cut-sets are inoperable simultaneously, then the system is inoperable during that time: ( $\mathrm{X}, \mathrm{Y}$ and $\mathrm{Z})$ or $(A$ and $B)$ or $(\mathrm{X}, \mathrm{Y}$ and $B)$ or $(\mathrm{Y}, \mathrm{Z}$ and $A)$, no other minimal combinations are fatal. In discrete event simulation, first random numbers are assigned to TTR and TBF of each component using the corresponding statistical distributions. Then every timeunit (s, min or h), TTR or TBF of each component is compared with the time from last change of the component's state and if necessary its state is changed. Meanwhile at every time-unit, state of the whole system is monitored using the predetermined minimum cut-sets. If system's previous state is not changed, then if it is working properly the TBF is incremented and if it is not, the TTR is increased by the unit time. In the case that the system's previous state is changed, if it was operable and now it is not, its TBF is saved and a timer is assigned to the TTR of the new state and if the system is now operable, its TTR should be saved and a new TBF timer is used for its new state. This process will continue for a period of time which is large relative to MTBF and MTTR of the components. For a large system, this approach would not be efficient because of the large number of failure cut-sets that would have to be determined manually.

In flow method you order (configure) the components in the simulation model as they are in the real system. You treat each component as a switch that is closed if the component is operable and open if it is not. Then every time-unit (s, min, or h), after determining states of the components, you send a pulse (transaction) through the system, splitting it into multiple directions when required. If this pulse reaches the output of the system, it is operable and else it is not. When state of the system is determined, TTR and TBF distributions are obtained just like failure mode method. The main 
International Journal of

Soft Computing And Software Engineering (JSCSE)

e-ISSN: 2251-7545

Vol.2,No.5, 2012
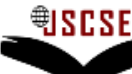

DOI: 10.7321/jscse.v2.n5.3

difference between this method and failure mode method is that here there is no need to determine minimum cut-sets of the system. In other words, this method does not require any failure mode identification and knowledge of the system/component configuration is sufficient.
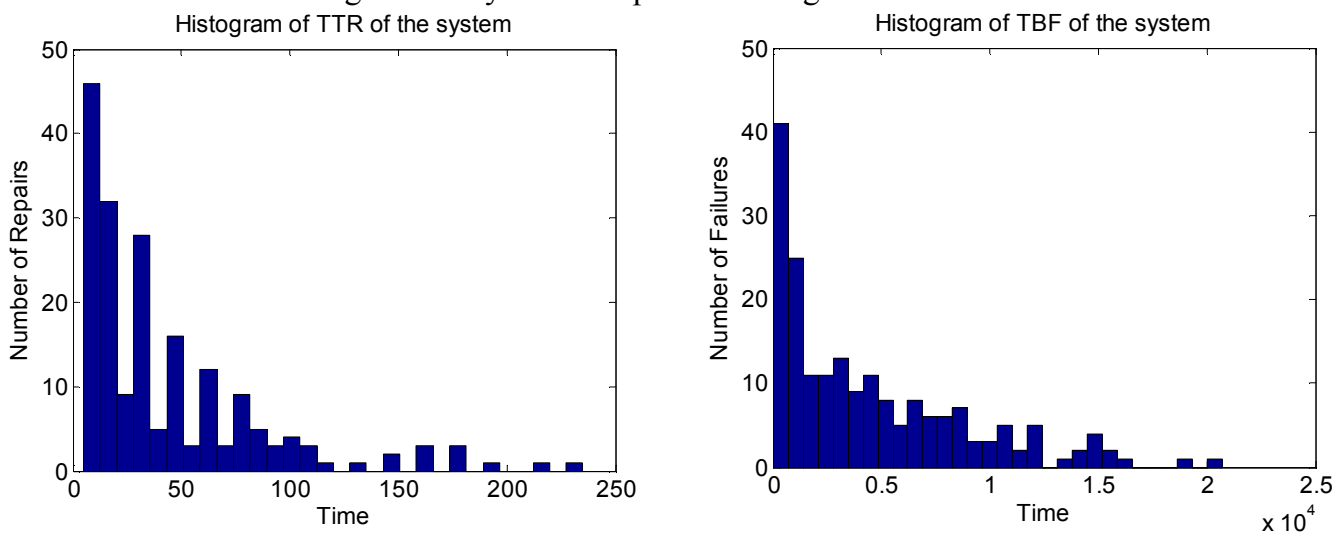

Figure 2. TBF and TTR histograms obtained trough simulation of 100 years of the system's operation.

We have used MATLAB to implement these methods. Histograms of the random variables TBF and TTR are shown in Figure 2 and CDF diagrams are plotted in Figure 3. These Figures show the result of simulating the system's operation for a period of 100 years. The simulation run time was about 7 minutes using a $2.8 \mathrm{GHz}$ CPU. It is clear that since the period of system's operation is not long enough, the size of collected data is small and the resulting diagrams are not smooth. To obtain more accurate estimates of TBF and TTR distributions, period of system's operation should be extended. However, this will take a long time of simulation run using the same CPU. This problem even is more critical if there are a large number of components in the system. In next section new methods are developed which will result in a great simulation time saving.

\section{Modified Approaches}

In order to reduce the amount of computations required, these methods are modified as follows: after generating a sequence of TBF and TTR values for the units, at each time-unit, state of every unit is observed in order to determine if it has changed or not. State of the system is determined only if at least state of one unit is changed (i.e. one unit is failed or repaired). Otherwise, after a time-unit, states of all units are observed again. This process is repeated until at least state of one unit is changed. When a change is detected, state of the system is determined using one of the methods of failure mode or flow. Finally, based on the system TBF and TTR values obtained, probability distributions of these random variables are estimated. The main innovation of these modified methods is that the system state is determined only when a component state is changed, where as in the conventional flow method and failure mode method the system state is monitored at each time-unit even if no change has occurred in the components' states. This innovation leads to a great time saving which is very important for large systems. Flowchart of modified flow method is illustrated in Figure 4. 

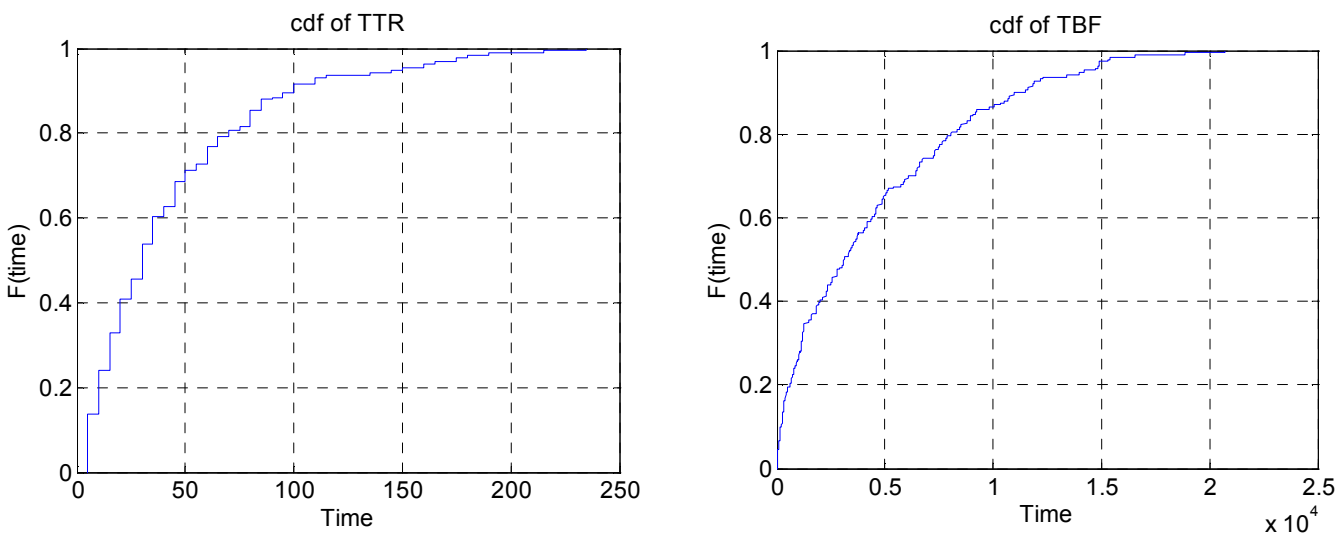

Figure 3. TBF and TTR CDFs obtained trough simulation of 100 years of the system's operation.

Reliability of the hydraulic network shown in Figure 1 is studied using these modified methods. Reduction of computational load allowed us to run Monte Carlo simulation for a long period of system operation and hence acquire an accurate estimate of TBF and TTR densities. It is important to note that this was impossible using previous methods. Using the modified flow method and a $2.8 \mathrm{GHz} \mathrm{CPU}$, simulation of 1000 years operation of the system took about 32 seconds. If it is compared with the previous approach (about 7 minutes for simulating 100 years operation of the system), the time saving is in the order of 0.01 . This is of great importance especially for large systems because the computation time required is proportional to number of units in the system.

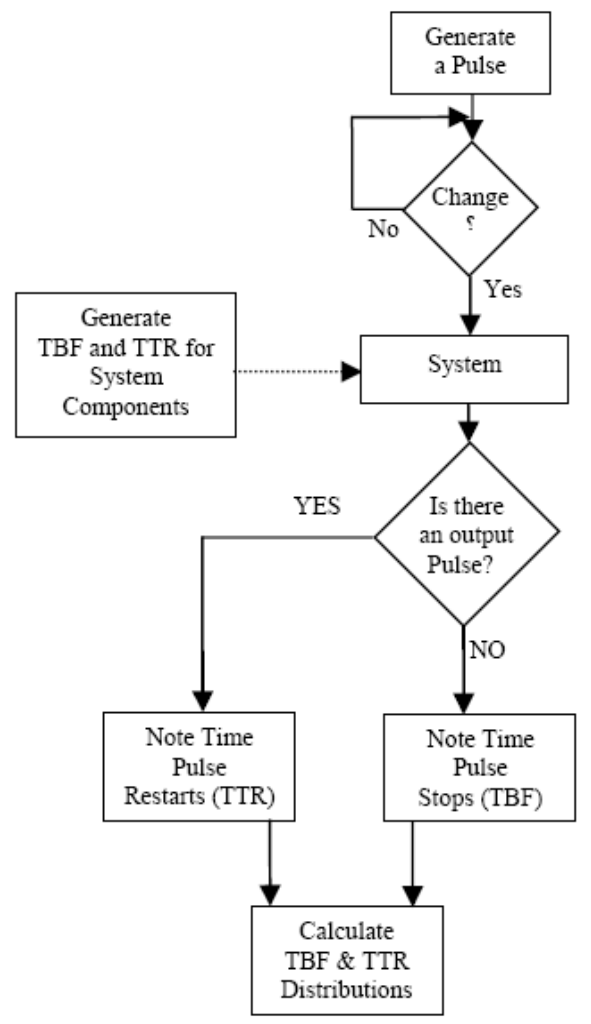

Figure 4. Modified flow method flowchart. 
International Journal of

Soft Computing And Software Engineering (JSCSE)

e-ISSN: 2251-7545

Vol.2,No.5, 2012

Operation of the hydraulic system is simulated for 1000 years and the results are illustrated in Figures 5 and 6. Histograms of the system TTR and TBF are shown in Figure 5 and CDF diagram of these variables are plotted in Figure 6.

As might have been expected, for this simple system, one can see that when TBF and TTR distributions are exponential for all units, the system TBF and TTR distributions will be exponential as well. For a more complex cross-linked system, exponentially distributed TBF and TTR for all components will not guarantee exponentially distributed TBF and TTR for the system.

If the component TTR distributions in Figure 1 are changed from exponential to normal distribution (with the same $M T T R=100 \mathrm{~h}$, but with $S_{T T R}=10$ ), a much different result occurs for this system as shown in Figures 7 and 8 . The system TBF distribution is exponential, as might have been expected however, the system TTR distribution does not have a standard form, which is surprising. None of this could be determined by analytical reliability analysis even for this simple example.

\section{Modified flow method modeling using Petri net}

In this section, a method for modeling modified flow method using PN is developed. Here the procedure is expressed through modeling the hydraulic network shown in Figure 1. This method is general and can be used for reliability analysis of any network.
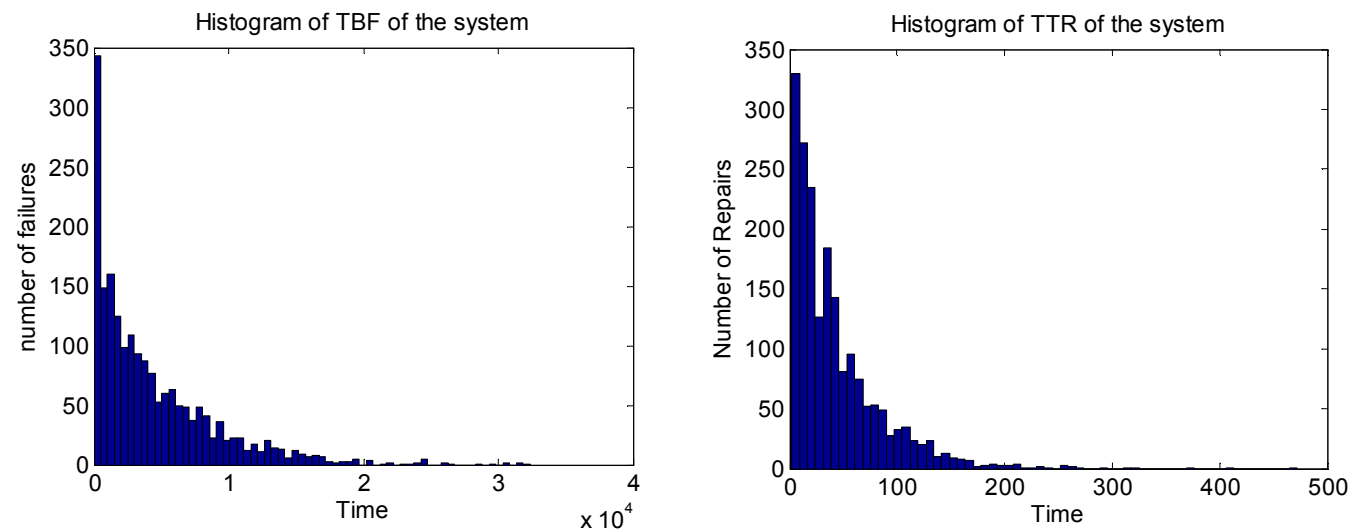

Figure 5. TBF and TTR histograms obtained trough simulation of 1000 years of the system's operation.
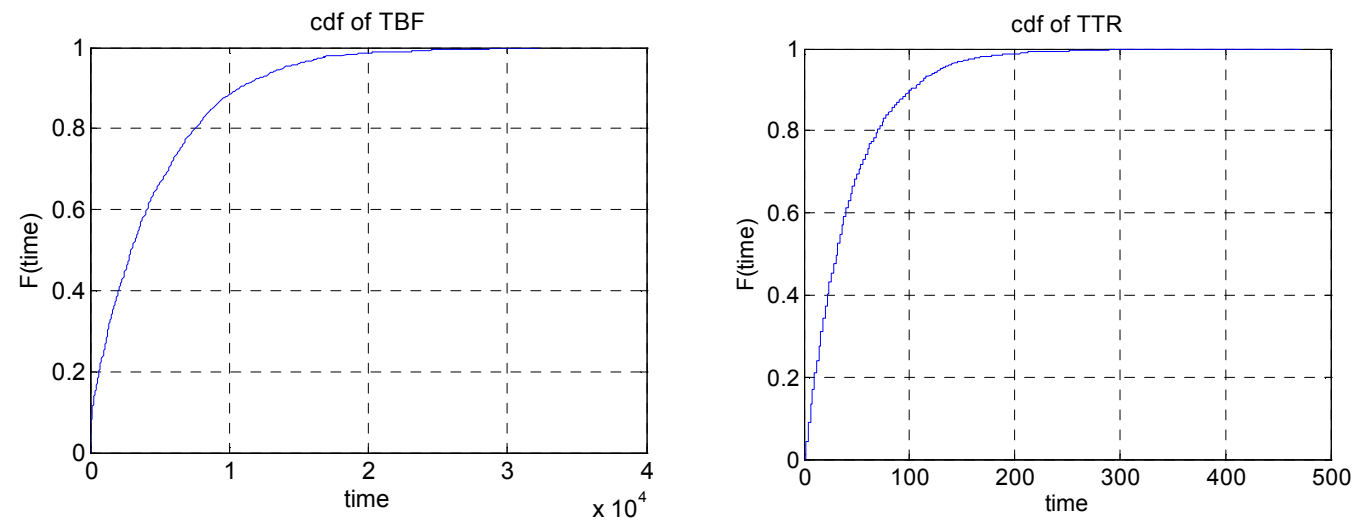

Figure 6. TBF and TTR CDFs obtained trough simulation of 1000 years of the system's operation. 

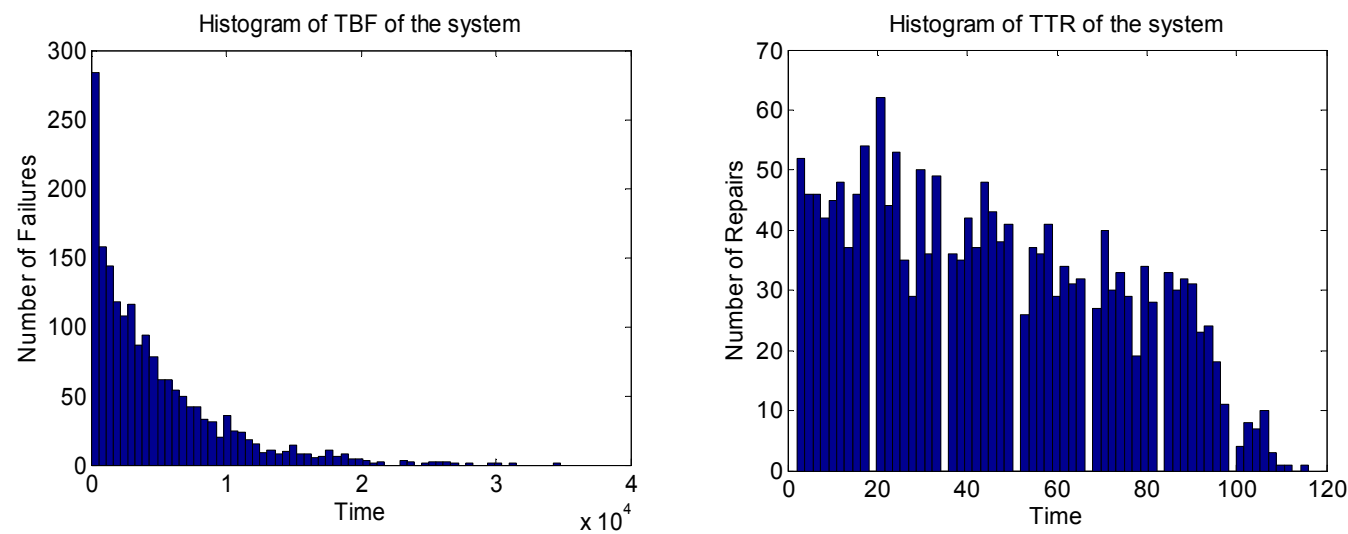

Figure 7. TBF and TTR histograms obtained trough simulation of 1000 years of the system's operation.

This PN is structured in two parts. The first part contains several Petri sub-nets, each of them formed by two places, two stochastic transitions, and four regular arcs. There is a Petri sub-net for every component of the system, as it is indicated in Figure 9. This first part of the Petri net is used to simulate the behavior of all components through possible states. The second part is used to determine whether the system works properly or not. This is done by simulating the flow of oil through pipes of the hydraulic network using Petri net modeling.

In this part, each component is modeled by a place. The transition from any place to any other place (from source to destination) is made through an immediate transition corresponding to an existing line. This transition has four arcs in correspondence with the following four conditions:

- the pump is functional - the place corresponding to repair state of pump is empty

- in the source place is a token

- destination place is empty (if it's not this node is already supplied)

- a change happened before (a pump was repaired or failed)

For the first and third conditions, inhibitor arcs and for the second condition regular arcs are used. For the fourth condition two places and an inhibitor arc are needed. These places are named CHANGE and MODE. The meaning of these two places is the following: after a change happens the CHANGE place receives one token. The next move is that both CHANGE and MODE places become empty.
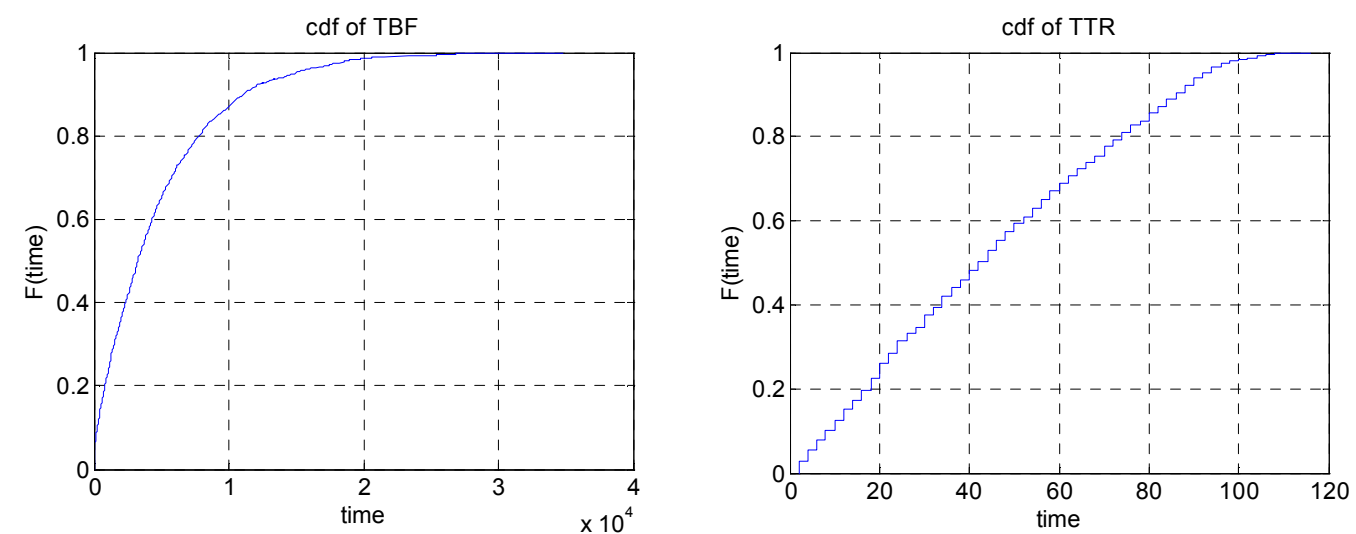

Figure 8. TBF and TTR CDFs obtained trough simulation of 1000 years of the system's operation. 
International Journal of

Soft Computing And Software Engineering (JSCSE)

e-ISSN: 2251-7545

Vol.2,No.5, 2012

From this point the function of Petri net is the following:

- all the places from the second part of Petri net get empty (all places from this part of net reset);

- in source node (NODE 1) a token is generated using an immediate transition which is conditioned by MODE place;

- the second part of Petri net begins to simulate the oil flow; this sequence is finished when all reachable nodes receive a token.

After all these operations, all immediate transitions are disabled and the stochastic transitions are enabled again and the network waits until something happens (failure or repair). During the time when stochastic transitions are enabled, the presence of a token in a place corresponding to a node of network means that node is supplied with oil. The PN network obtained through this modeling method is shown in Figure 9.

The network reliability modeling technique introduced here has two main advantages: First, since Petri net is a standard tool for modeling discrete event systems, using this technique, one can implement the modified flow method through a systematic and standard approach. Software tools developed for PN modeling can also be used for this purpose. Second, since the developed model is simple and graphical, it would greatly help solving the reliability analysis problem. This graphical model will simplify writing and debugging of the required codes and gives a feeling of how failure of each component will affect operation of the whole system.

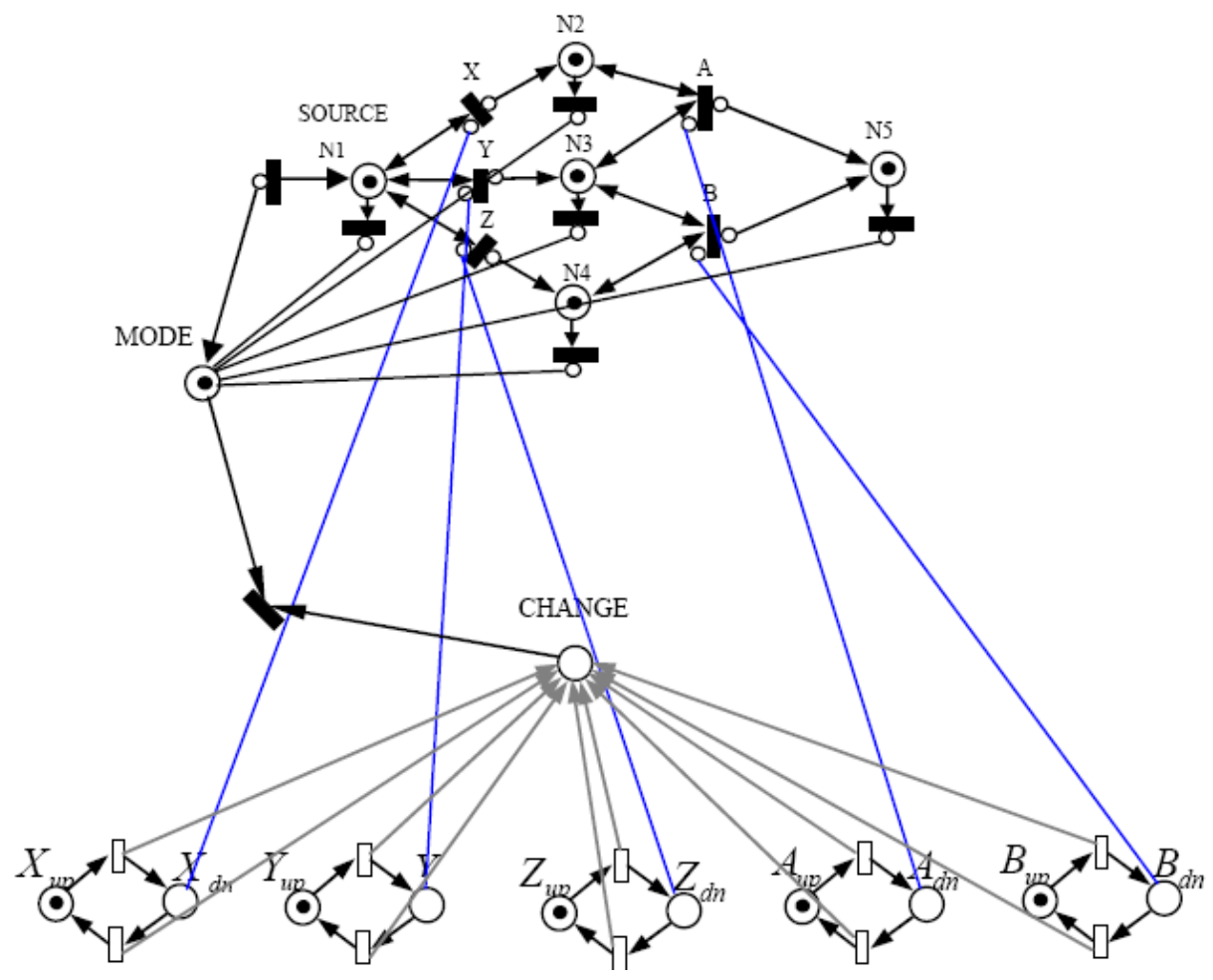

Figure 9. The Petri Net Model for the proposed network (depicted in Figure 1).

\section{Conclusion}

Failure-mode method and flow method are two general approaches through which reliability of any 
International Journal of

Soft Computing And Software Engineering (JSCSE)

e-ISSN: 2251-7545

Vol.2,No.5, 2012

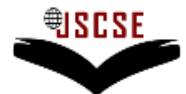

DOI: 10.7321/jscse.v2.n5.3

complex network can be analyzed. In this paper these methods were further explored and applied to a typical hydraulic network. In fact writing a code for failure-mode method is simpler but one should determine failure modes of the system beforehand which is a laborious job. Flow method does not need this failure mode identification but the system's structure and components' interconnections must be implemented directly by the code written to simulate the system's operation. These methods were modified to make discrete event simulation approach more efficient and reduce computational load. Since required computations is proportional to number of system's components, for a large complex system, this modification will lead to a great time saving and therefore much more exact results can be obtained by decreasing the time-unit and increasing the period of system operation time which is simulated.

Petri net is a powerful modeling tool for discrete event systems. Since failure and repair of a system's components are events which may occur according to known statistical distributions, these events can be modeled using Petri net and stochastic Petri net. Therefore one can use techniques and softwares developed for Petri net networks in reliability analysis. In this paper a PN modeling approach is developed for the modified flow method and it is shown how this model can be used to analyze reliability of the typical hydraulic network. This PN modeling approach makes reliability analysis problem simpler and its graphical representation helps determining role of each component failure in operation of the whole system.

Our proposed discrete-event modeling and simulation method can be used for reliability analysis of any systems, without any limitation on the components configuration or system scale. The only limiting factor might be the computational capabilities of the simulating computers.

The Petri net approach seems to be a strong method of reliability analysis, therefore its applications in reliability assessment can be studied more. Furthermore, the proposed method's output is some data points (some particles) indicating the TTR and TBF distribution, however some statistical analysis tools might be used for modeling of data by a smooth function. Wavelet functions have been previously used for distribution modeling so their application in reliability analysis might be considered. The above mentioned points are some open research issues that can be studied in future works.

\section{References}

[1] A. Hoyland, M. Pausand, System Reliability Theory, Models and Statistical Methods. John Wiley \& Sons Inc., 1994.

[2] B. Gnedenko, I. Pavlov, I. Ushakov, Statistical Reliability Engineering. John Wiley \& Sons Inc., 1999.

[3] E. E. Lewis, Introduction to Reliability Engineering. Wily, 1987.

[4] I. Bazovsky, Reliability Theory and Practice. Prentice-Hall, 1961.

[5] E. J. Henley, H. Kumamoto, Reliability Engineering and Risk Assessment. Prentice-Hall, 1981.

[6] Y. Chun-man, G. Bao-long, W. Xian-xiang, "Empirical Study of the Inertia Weight Particle Swarm Optimization with Constraint Factor," International Journal of Soft Computing and Software Engineering, vol. 2, no. 2, pp. 1-8, 2012.

[7] J. A. Chisman, "Using Discrete Simulation Modeling to Study Large-Scale System Reliability/Availability," Computers Ops Res., vol. 25, no. 3, pp. 169-174, 1998.

[8] D. C. Ionescu, A. P. Ulmeanu, "Efficient computational techniques for power systems reliability assessment,"

[9] K. Jensen, G. Rozenberg, High-level Petri Nets. Theory and Application. Springer-Verlag, 1991.

[10] S. Christensen, K. Jensen, T. Mailund, "State Space Methods for Timed Petri Nets",Proc. of 2nd Int. Coll. on Petri Net Technologies for Modelling Communication Based Systems, pp. 33-42, 2001. 
International Journal of

Soft Computing And Software Engineering (JSCSE)

e-ISSN: 2251-7545

Vol.2,No.5, 2012

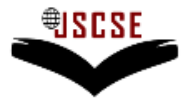

DOI: 10.7321/jscse.v2.n5.3

[11] A.P. Ulmeanu, D.C. Ionescu, "The Computer-Assisted Analysis of the semi-Markovian Stochastic Petri Nets and an Application," Statistical and Probabilistic Models in Reliability, pp. 307-320. Boston: Birkhauser, 1999.

[12] Y. Jiang, Z. Qiu, J. Zhang, J. Li, "Integration of Unicast and Multicast Scheduling Input-Queued Pachet Switches with High Scalability, " International Journal of Soft Computing and Software Engineering, vol. 2, no. 4, pp. 14-25, 2012.

[13] M. P. Cabasino, A. Giua, M. Pocci, C. Seatzu, "Discrete event diagnosis using labeled Petri nets. An application to manufacturing systems," Control Engineering Practice, vol. 19, pp. 989-1001, 2011.

[14] O. Nyvlt, M. Rausnad, "Dependencies in event trees analyzed by Petri nets," Reliability Engineering and System Safety, vol. 104, pp. 45-57, 2012. 